\title{
DISCONJUGACY AND OSCILLATION OF THIRD ORDER DIFFERENTIAL EQUATIONS WITH NONNEGATIVE COEFFICIENTS
}

\author{
G. J. ETGEN AND C. D. SHIH
}

\begin{abstract}
The purpose of this paper is to establish conditions which imply that the third order linear differential equation with nonnegative coefficients defined on an infinite interval will fail to be disconjugate on any infinite subinterval. Assuming that the equation is not disconjugate on any infinite subinterval, conditions are presented which establish that the equation has oscillatory solutions. These results are in partial answer to questions raised by J. H. Barrett. The oscillation criteria obtained here are similar to the oscillation conditions established by A. C. Lazer.
\end{abstract}

1. Introduction. The relationship between disconjugacy and oscillation for second order linear differential equations is an easy consequence of the Sturm separation theorem. In particular, the equation

$$
\left(p(x) y^{\prime}\right)^{\prime}+q(x) y=0 \text { on }[a, \infty),
$$

where $p(x)$ and $q(x)$ are continuous functions and $p(x)>0$, is nonoscillatory on $[a, \infty)$ if and only if there exists a number $b, b \geqq a$, such that (1) is disconjugate, i.e., no nontrivial solution of (1) has more than one zero, on $[b, \infty)$. Alternatively, (1) is oscillatory on $[a, \infty)$ if and only if for each $c \in[a, \infty)$ there exists a nontrivial solution of (1) having at least two zeros on $[c, \infty)$.

In [1], J. H. Barrett studied the third order linear differential equation

$$
y^{\prime \prime \prime}+p(x) y^{\prime}+q(x) y=0 \text { on }[a, \infty),
$$

where $p(x)$ and $q(x)$ are continuous functions on $[a, \infty), p(x) \geqq 0, q(x) \geqq 0$ and $p(x)+q(x) \not \equiv 0$ on any subinterval of $[a, \infty)$. The objective of his paper was to establish conditions which would insure that for each number $c \in[a, \infty)$, there exists a nontrivial solution of (2) having at least three zeros on $[c, \infty)$, i.e., that (2) is not disconjugate on $[c, \infty)$ for any number $c \in[a, \infty)$. His paper, unfortunately, contained two mistakes which invalidated his key theorem:

Received by the editors March 7, 1972 and, in revised form, August 16, 1972.

AMS (MOS) subject classifications (1970). Primary 34C10.

Key words and phrases. Third order linear differential equations, disconjugacy, oscillation of solutions.

(c) American Mathematical Society 1973 
THEOREM 1 [1, THEOREM 4.1]. If (2) is disconjugate on $[a, \infty)$ and $u(x, a)$ is the solution of (2) satisfying the initial condition

$$
y(a)=y^{\prime}(a)=0, \quad y^{\prime \prime}(a)=1,
$$

then $u^{\prime \prime}(x, a)>0$ on $[a, \infty)$. Moreover $u(x, a)>0, \quad u^{\prime}(x, a)>0$ and $u^{\prime \prime \prime}(x, a)<0$ on $(a, \infty)$.

Using Barrett's techniques, the authors [3] obtained Theorem 1 when the coefficients are assumed to satisfy the stronger hypotheses:

$$
\begin{aligned}
& p(x) \geqq 0, q(x)>0, \quad \text { on }[a, \infty), \\
& p(x) / q(x) \text { nondecreasing on }[a, \infty) .
\end{aligned}
$$

A similar result was obtained by A. C. Lazer [5, Lemma 3.1] using hypotheses: $p(x) \in C^{\prime}[a, \infty), p(x) \geqq 0, q(x) \geqq 0$ and $2 q(x)-p^{\prime}(x) \geqq 0$ on $[a, \infty)$ with $2 q(x)-p^{\prime}(x)$ not identically zero on any subinterval. Lazer uses his result as a basic tool in the development of oscillation and nonoscillation criteria for (2) [5, Theorems 3.1 and 3.5].

Assuming that the coefficients of $(2)$ satisfy $(\mathrm{H})$, the following theorem is a consequence of Theorem 1 .

THEOREM 2. If either of the second order equations

or

$$
y^{\prime \prime}+\left[p(x)+\frac{1}{2}(x-a) q(x)\right] y=0
$$

$$
y^{\prime \prime}+\left[p(x)+\int_{x}^{\infty} q(t) d t\right] y=0
$$

is oscillatory on $[a, \infty)$, then there is a nontrivial solution of (1) having at least three zeros on $[a, \infty)$. (See [1, §5] and [3].)

The purpose of this paper is to establish conditions which will imply that (2) is not disconjugate on any subinterval $[c, \infty)$ of $[a, \infty)$ and to present certain cases where the nondisconjugacy of $(2)$ on $[c, \infty)$ for every $c \in[a, \infty)$ actually implies the oscillation of (2). These results, then, partially answer the question raised by Barrett [1] on the relation between disconjugacy and oscillation for third order equations. There are examples of third order equations which are not disconjugate on any subinterval $[c, \infty)$ of $[a, \infty)$ and yet are nonoscillatory (cf. [1, p. 213]) in contrast to the situation with second order equations.

The results in $\S \S 2$ and 3 may be combined to give oscillation criteria for (2) which are similar to the oscillation condition established by Lazer [5, Theorem 3.1]. However, our approach through nondisconjugacy on any subinterval is different from the techniques used by Lazer in $[5, \S 3]$. 
2. Disconjugacy. In this section we establish conditions which will imply that for each number $c \in[a, \infty)$ there will exist a nontrivial solution of (2) having at least three zeros on $[c, \infty)$. Our first theorem follows from the methods described in [1, $\S 5$, pp. 220 and 221] together with the authors' work in [3].

THEOREM 3. Let the coefficients of (2) satisfy (H). If the second order equation

$$
y^{\prime \prime}+\left[p(x)+\frac{1}{2}(x-c) q(x)\right] y=0
$$

is oscillatory on $[a, \infty)$ for each $c \in[a, \infty)$, or if the second order equation

$$
y^{\prime \prime}+\left[p(x)+\int_{x}^{\infty} q(t) d t\right] y=0
$$

is oscillatory on $[a, \infty)$, then for each $c \in[a, \infty)$ there is a nontrivial solution $y(x)$ of $(2)$ having at least three zeros on $[c, \infty)$.

Proof. Suppose there exists a number $\bar{c} \in[a, \infty)$ such that no nontrivial solution of (2) has more than two zeros on $[\bar{c}, \infty)$. Then (2) is disconjugate on $[\bar{c}, \infty)$ and the solution $u(x, \bar{c})$ satisfying the initial conditions

$$
y(\bar{c})=y^{\prime}(\bar{c})=0, \quad y^{\prime \prime}(\bar{c})=1
$$

has the property $u(x, \bar{c})>0, u^{\prime}(x, \bar{c})>0, u^{\prime \prime}(x, \bar{c})>0, u^{\prime \prime \prime}(x, \bar{c})<0$ on $(\bar{c}, \infty)[3$, Theorem 1]. Thus the second order equation

$$
y^{\prime \prime}+\left[p(x)+\left(u(x, \bar{c}) / u^{\prime}(x, \bar{c})\right) q(x)\right] y=0
$$

is satisfied by the positive solution $u^{\prime}(x, \bar{c})$ on $(\bar{c}, \infty)$. Using a result of Lazer [5, Lemma 3.2], it follows that

$$
u(x, \bar{c}) / u^{\prime}(x, \bar{c}) \geqq(x-\bar{c}) / 2 .
$$

Therefore, by the Sturm comparison theorem, the second order equation

$$
y^{\prime \prime}+\left[p(x)+\frac{1}{2}(x-\bar{c}) q(x)\right] y=0
$$

is disconjugate on $[\bar{c}, \infty)$, contradicting our first hypothesis.

Barrett's argument $[1$, p. 221] establishes that the oscillation of (4) implies the conclusion of the theorem.

We note that since $q(x)>0$ on $[a, \infty)$, if the second order equation

$$
y^{\prime \prime}+p(x) y=0
$$

is oscillatory on $[a, \infty)$, then the Sturm comparison theorem yields the fact that (3) is oscillatory on $[a, \infty)$ for each $c \in[a, \infty)$ and (4) is oscillatory on $[a, \infty)$. Of course either (3) or (4) may be oscillatory without (5) being oscillatory. 
The proof of our next theorem requires the following well-known fact.

LEMMA. If each of the second order equations

$$
y^{\prime \prime}+p_{i}(x) y=0, \quad i=1,2, \cdots, n,
$$

is disconjugate on $[a, \infty)$ and $c_{1}, c_{2}, \cdots, c_{n}$ are nonnegative constants such that $\sum_{1}^{n} c_{i}=1$, then the equation

is disconjugate on $[a, \infty)$.

$$
y^{\prime \prime}+\left(\sum_{1}^{n} c_{i} p_{i}\right) y=0
$$

THEOREM 4. Let the coefficients of (2) satisfy (H). If there exists a number $m, m<\frac{1}{2}$, such that the second order equation

$$
y^{\prime \prime}+[p(x)+m(x-a) q(x)] y=0
$$

is oscillatory on $[a, \infty)$, then for each $c \in[a, \infty)$, there is a nontrivial solution $y(x)$ of $(2)$ having at least three zeros on $[c, \infty)$.

Proof. We note first that if (7) is oscillatory on $[a, \infty)$ for some $m \leqq 0$, then $(5)$ is oscillatory on $[a, \infty)$ since $q(x)>0$. Thus the conclusion of our theorem follows from our remark at the end of the proof of Theorem 3.

Let $m, 0<m<\frac{1}{2}$, be a number such that (7) is oscillatory and suppose there exists a number $\bar{c} \in[a, \infty)$ such that no nontrivial solution of (2) has more than two zeros on $[\bar{c}, \infty)$. Then as in the proof of Theorem 3 , the second order equation

$$
y^{\prime \prime}+\left[p(x)+\frac{1}{2}(x-\bar{c}) q(x)\right] y=0
$$

is disconjugate on $[\bar{c}, \infty)$. Clearly, $(8)$ is disconjugate on $[b, \infty)$ for all $b \geqq \bar{c}$. Define $\varepsilon, \varepsilon>0$, by the equation $m=1 /(2+\varepsilon)$ and let $N$ be a positive number such that $1 / N<\varepsilon$. By the Sturm comparison theorem, the second order equation

$$
y^{\prime \prime}+[p(x)+N(\bar{c}-a) q(x)] y=0
$$

is disconjugate on $[2 N(\bar{c}-a)+\bar{c}, \infty)$ since $p(x)+\frac{1}{2}(x-\bar{c}) q(x) \geqq p(x)+$ $N(\bar{c}-a) q(x)$ on this interval. Multiplying (8) by $2 N /(2 N+1)$, (9) by $1 /(2 N+1)$, and adding, yields the equation

$$
y^{\prime \prime}+[p(x)+(N /(2 N+1))(x-a) q(x)] y=0
$$

which, by the lemma, is disconjugate on $[2 N(\bar{c}-a)+\bar{c}, \infty)$, where $2 N(\bar{c}-a)+\bar{c} \geqq \bar{c} \geqq a$. Now

$$
\begin{array}{r}
p(x)+(1 /(2+1 / N))(x-a) q(x)>p(x)+(1 /(2+\varepsilon))(x-a) q(x) \\
=p(x)+m(x-a) q(x)
\end{array}
$$


and we conclude that (7) is disconjugate on $[2 N(\bar{c}-a)+\bar{c}, \infty)$ which contradicts the hypothesis of the theorem.

Summarizing the results of this section: if the coefficients of (2) satisfy $(\mathrm{H})$, then each of the following is a sufficient condition that (2) fail to be disconjugate on each subinterval $[c, \infty)$ of $[a, \infty)$.

(i) $y^{\prime \prime}+p(x) y=0$ is oscillatory on $[a, \infty)$,

(ii) $y^{\prime \prime}+\left[p(x)+\frac{1}{2}(x-c) q(x)\right] y=0$ is oscillatory on $[a, \infty)$ for each $c \in[a, \infty)$,

(iii) $y^{\prime \prime}+\left[p(x)+\int_{x}^{\infty} q(t) d t\right] y=0$ is oscillatory on $[a, \infty)$,

(iv) there exists a number $m, m<\frac{1}{2}$, such that

$$
y^{\prime \prime}+[p(x)+m(x-a) q(x)] y=0
$$

is oscillatory on $[a, \infty)$.

Condition (i) implies each of the other three conditions. Conditions (ii) and (iii) are independent (cf. [1, p. 213]).

3. Oscillation. In this section we shall assume that (2) has the property that for each number $c \in[a, \infty)$ there exists a nontrivial solution of (2) having at least three zeros on $[c, \infty)$. We consider the question of establishing that this property of (2) implies the existence of a nontrivial solution of (2) having infinitely many zeros on $[a, \infty)$. Our result here is merely a combination of the work of M. Hanan [4] and Barrett [2, $\S \mathrm{II}]$.

THEOREM 5. If (2) is not disconjugate on any subinterval $[c, \infty)$ of $[a, \infty)$, then each of the following is a sufficient condition that (2) have a nontrivial oscillatory solution:

(a) $y^{\prime \prime}+p(x) y=0$ is nonoscillatory on $[a, \infty)$,

(b) $p(x) \in C^{\prime}[a, \infty)$ with $2 q(x)-p^{\prime}(x)$ of one sign on $[a, \infty)$ and not identically zero on any subinterval.

Proof. According to Hanan [4], if (a) holds, then (2) is of class $C_{I}$ [4, Theorem 2.3]. If (b) holds and $2 q(x)-p^{\prime}(x) \geqq 0(\leqq 0)$ on $[a, \infty)$, then (2) is of class $C_{\mathrm{I}}\left(C_{\mathrm{II}}\right)$ [4, Theorem 2.2].

Suppose that (2) is nonoscillatory. Since (2) is not disconjugate on any subinterval $[c, \infty)$ of $[a, \infty)$, the adjoint

$$
y^{\prime \prime \prime}+p(x) y^{\prime}+\left(p^{\prime}(x)-q(x)\right) y=0
$$

of (2) has oscillatory solutions [2, Theorem 2.15]. Referring again to Hanan's work [4, Theorem 4.7], we conclude that the oscillation of (11) implies that (2) has oscillatory solutions, contradicting our supposition and completing the proof. 
The conditions (i)-(iv) at the end of $\$ 2$ can be combined with conditions (a) or (b) above to give various oscillation criteria for (2). For example, an oscillation condition which is analogous to Lazer's result [5, Theorem 3.1 ] is obtained by combining conditions (iv) and (b). We have:

THEOREM 6. Let the coefficients of (2) satisfy (H). If there exists a number $m, m<\frac{1}{2}$, such that (7) is oscillatory on $[a, \infty)$, and if $p(x) \in$ $C^{\prime}[a, \infty)$ with $2 q(x)-p^{\prime}(x)$ of one sign on $[a, \infty)$ and not identically zero on any subinterval, then (2) has oscillatory solutions.

\section{REFERENCES}

1. J. H. Barrett, Third order differential equations with non-negative coefficients, J. Math. Anal. Appl. 24 (1968), 212-224. MR 38 \#365.

2. - Oscillation theory of ordinary linear differential equations, Advances in Math. 3 (1969), 415-509. MR 41 \#2113.

3. G. J. Etgen and C. D. Shih, Disconjugacy of third order differential equations with non-negative coefficients, J. Math. Anal. Appl. (to appear).

4. M. Hanan, Oscillation criteria for third-order differential equations, Pacific J. Math. 11 (1961), 919-944. MR 26 \#2695.

5. A. C. Lazer, The behavior of solutions of the differential equation $y^{\prime \prime \prime}+p(x) y^{\prime}+$ $q(x) y=0$, Pacific J. Math. 17 (1966), 435-466. MR 33 \#1552.

Department of Mathematics, University of Houston, Houston, TeXas 77004 